\title{
Feline Lentivirus Group
}

National Cancer Institute

\section{Source}

National Cancer Institute. Feline Lentivirus Group. NCI Thesaurus. Code C117714.

A non-taxonomic grouping of lentivuruses that infect lions, tigers, jaguars, and other wild and domestic cats. 